Anaesthesist 2010 · 59:255

DOI 10.1007/s00101-010-1697-7

๑) Springer-Verlag 2010
E.-M. Jordi-Ritz

Abteilung für Anästhesie, Universitätskinderklinik beider Basel
Jordi Ritz E-M, Fich C, Gisin S et al (2009)

Anaesthesist 58:1231-1238

Im oben genannten Beitrag wurde die - Abb. 4 leider fehlerhaft wiedergegeben.

SEKT - steht für Situationsbewusstsein, Entscheidungsfindung, Kommunikation und Teamarbeit. Es handelt sich dabei um die nichttechnischen Fertigkeiten, die durch Simulationstrainings

\title{
Erratum zu: Kindersimulation heute und morgen - Perspektiven und Konzepte
}

positiv und nachhaltig beeinflusst werden können.

Gerade in jener $\bullet$ Abb. 4, die diese nichttechnischen Fertigkeiten beschreibt, ist ein für das Verständnis des Beitrags relevanter Fehler zu finden. To err is human. In diesem Sinne bitten wir den aufmerksamen Leser um Nachsicht.

Aus technischen Gründen kann die ursprüngliche Abb. 4 im Erratum nur als - Abb. 1 wiedergegeben werden.

\section{Korrespondenzadresse}

Dr. E.-M. Jordi-Ritz

Abteilung für Anästhesie, Universitätskinderklinik beider Basel

Römergasse 8, 4005 Basel

Schweiz

EvaMaria.Jordi@ukbb.ch

Die Online-Version des Originalartikels können Sie unter http://dx.doi.org/10.1007/s00101-0091642-9 finden.
Abb. $1>$ Merkhilfe für die Beobachtung nichttechnischer Fertigkeiten: SEKT (Situationsbewusstsein,

Entscheidungsfindung, Kommunikation, Teamarbeit). (Mod. Beurteilungssystem nach [7])

\begin{tabular}{|c|c|}
\hline & $\begin{array}{l}\text { Arbeitsumgebung kennen } \\
\text { Informationen erkennen und sammeln } \\
\text { Informationen interpretieren } \\
\text { Probleme antizipieren } \\
\text { Änderungen bemerken } \\
\text { Informationen erkennen + sammeln }\end{array}$ \\
\hline & $\begin{array}{l}\text { Probleme definieren } \\
\text { Optionen darlegen } \\
\text { Vorgehen planen } \\
\text { Prioritäten setzen } \\
\text { Überprüfen der Resultate }\end{array}$ \\
\hline & $\begin{array}{l}\text { Sichere und effiziente Kommunikation } \\
\text { Informationsaustausch im Kontext } \\
\text { Feedback geben } \\
\text { Respektvoller Umgang }\end{array}$ \\
\hline & $\begin{array}{l}\text { Konsensbildung über die Situation } \\
\text { Gemeinsames Ziel definieren } \\
\text { Koordination und Aufteilung der Arbeit } \\
\text { Führungsfunktion übernehmen } \\
\text { Ressourcen erkennen und Hilfe anfordern } \\
\text { Andere unterstützen }\end{array}$ \\
\hline
\end{tabular}

SituationsbewusstseinEntscheidungsfindungKommunikationTeamarbeit * Modifiziertes Beurteilungssystem nach G. Fletcher und R. Flin 